\title{
COMPLETE CHARACTERIZATIONS OF WEAKLY Po AND RELATED SPACES AND PROPERTIES
}

\section{CHARLES DORSETT}

Department of Mathematics

Texas A\&M University-Commerce

Texas 75429

USA

e-mail: charles.dorsett@tamuc.edu

\begin{abstract}
In 1936, $T_{0}$-identification spaces were introduced. In 2015, $T_{0}$-identification spaces were used to define weakly $P o$ spaces and properties and $T_{0}$-identification $P$ properties. Within this paper, complete characterizations for each of $T_{0}$-identification space properties, weakly $P o$ spaces and properties, and $T_{0}$-identification $P$ properties are given revealing new basic, never before imagined properties and relationships within topology.
\end{abstract}

\section{Introduction and Preliminaries}

$T_{0}$-identification spaces were introduced in 1936 [12].

Definition 1.1. Let $(X, T)$ be a space, let $R$ be the equivalence relation on $X$ defined by $x R y$ iff $C l(\{x\})=C l(\{y\})$, let $X_{0}$ be the set of $R$ equivalence classes of $X$, let $N: X \rightarrow X_{0}$ be the natural map, and let 2010 Mathematics Subject Classification: 54B15, 54D10, 54D15.

Keywords and phrases: $T_{0}$-identification spaces, topological properties, weakly Po.

Received May 22, 2017; Revised May 24, 2017

(ㄷ) 2017 Scientific Advances Publishers 
$Q(X, T)$ be the decomposition topology on $X_{0}$ determined by $(X, T)$ and the natural map $N$. Then $\left(X_{0}, Q(X, T)\right)$ is the $T_{0}$-identification space of $(X, T)$.

Within a 1936 paper [12], $T_{0}$-identification spaces were used to further characterize metrizable spaces: A space is pseudometrizable iff its $T_{0}$-identification space is metrizable.

In the 1975 paper [11], the $R_{1}$ property and $T_{0}$-identification spaces were used to further characterize the $T_{2}$ property.

Definition 1.2. A space $(X, T)$ is $R_{1}$ iff for $x$ and $y$ in $X$ such that $C l(\{x\}) \neq C l(\{y\})$, there exist disjoint open sets $U$ and $V$ such that $x \in U$ and $y \in V[1]$.

Theorem 1.1. A space is $R_{1}$ iff its $T_{0}$-identification space is $T_{2}$ [11].

Since for any topological property $P$ and any space with property $P$, its $T_{0}$-identification space exists, then there are no restrictions on spaces for which its $T_{0}$-identification space exists. Thus attention shifts from properties of spaces $(X, T)$ for which its $T_{0}$-identification space $\left(X_{0}, Q(X, T)\right)$ exists to the properties of the $T_{0}$-identification spaces $\left(X_{0}, Q(X, T)\right)$ motivating the definition and work below.

Definition 1.3. A topological property $P$ is a $T_{0}$-identification space property iff there exists a space $(X, T)$, whose $T_{0}$-identification space has property $P$.

In the 1936 paper [12], it was shown that $T_{0}$-identification spaces satisfy the $T_{0}$ separation axiom. Thus, for a topological property to be a $T_{0}$-identification space property, $\left(P\right.$ and $T_{0}$ ), denoted by $P o$, would have to exist. Within a 2007 paper [2], it was shown that a space is $T_{0}$ iff the natural map $N$ from the space onto its $T_{0}$-identification space is a homeomorphism. Thus, for each topological property $P$ for which $P o$ exists, $P o$ is a $T_{0}$-identification space property. 
Within a 1977 paper [3], several topological properties, including $R_{1}$, were shown to be simultaneously shared by a space and its $T_{0}$-identification space. Thus $R_{1}$ is a $T_{0}$-identification space property that is not $T_{0}$, raising the question of precisely which topological properties are $T_{0}$-identification space properties.

In the 2015 paper [4], the characterizations of metrizable and $T_{2}$ given above motivated the introduction and investigation of weakly $P_{0}$ spaces and properties.

Definition 1.4. Let $P$ be a topological property for which $P o$ exists. Then a space $(X, T)$ is weakly $P o$ iff its $T_{0}$-identification space $\left(X_{0}, Q(X, T)\right)$ has property $P$. A topological property $Q o$ for which weakly $Q o$ exists is called a weakly Po property.

Since the $T_{0}$-identification space of each space is $T_{0}$, then for a topological property $Q$ for which weakly $Q o$ exists, a space $(X, T)$ is weakly $Q o$ iff $\left(X_{0}, Q(X, T)\right)$ has property $Q o$, and, within $\left(X_{0}, Q(X, T)\right)$, $Q$ and $Q o$ are equivalent.

In the 2015 paper [4], it was shown that $R_{1}=\operatorname{weakly}\left(R_{1}\right) o=$ weakly $T_{2}$ and pseudometrizable $=$ weakly (pseudometizable) $o=$ weakly (metrizable). Hence $R_{1}$ and pseudometrizable are weakly $P_{o}$, and $T_{2}$ and metrizable are weakly Po properties. Also, in the 2015 paper [4], it was shown that for a topological property $Q$ for which weakly $Q o$ exists, weakly $Q_{o}$ is simultaneously shared by both a space and its $T_{0}$-identification space, which when combined with the results above, led to the introduction and investigation of $T_{0}$-identification $P$ properties.

Definition 1.5. A topological property $S$ is a $T_{0}$-identification $P$ property iff $S$ is simultaneously shared by both a space and its $T_{0}$-identification space [5]. 
In the 2015 paper [5], it was shown that for a $T_{0}$-identification $P$ property $S, S$ = weakly $S o$. Combining this result with the result above concerning weakly $P o$, the $T_{0}$-identification $P$ properties and the properties that are weakly $P o$ are exactly the same. Since for a space $(X, T)$ which is only one of $T_{0}$ and "not- $T_{0}$ ", $\left(X_{0}, Q(X, T)\right)$ is $T_{0}$, then neither $T_{0}$ nor "not- $T_{0}$ " are weakly $P o$ and there are restrictions on topological properties for which weakly Po exists.

The study of weakly $P o$ spaces and properties has been a fruitful study revealing the importance of the long-neglected topological property "not- $P$ " in the study of topology, where $P$ is a topological property for which "not- $P$ " exists. Thus far, the addition and use of "not- $P$ " in the study of topology has led to the discovery of the never before imagined least of all topological properties $L=\left(T_{0}\right.$ or "not- $T_{0}$ ") [6] and that there is no strongest topological property [7]. As is expected, the existence of the never before imagined topological property $L$ revealed needed changes for product [8] and subspace properties [9] leading to new, meaningful, never before imagined properties and examples for each of those two properties, expanding and changing the study of topology forever.

Below, each of the $T_{0}$-identification space properties, the topological properties that are weakly $P o$, and the topological properties that are weakly $P o$ properties are completely characterized revealing additional basic, fundamental, foundational, never before imagined properties and relationships in the area of topology. 


\section{The Complete Characterization of the Weakly Po Properties}

Let $n$ be a natural number, $n \geq 2$.

Definition 2.1. Let $Q$ be a topological property for which $Q o$ exists. A space $(X, T)$ is $Q(1, n)$ iff there exist $n$ distinct elements $a_{1}, \cdots, a_{n}$ all of whose closures are equal, and for all other $x \in X, C l(\{x\})=\operatorname{Cl}(\{y\})$ iff $x=y$, and the $T_{0}$-identification space of $(X, T)$ is $Q o$.

Below the existence of $Q(1, n)$ is established.

Theorem 2.1. Let $f: X \rightarrow Y$. Then $f$ is onto iff for each $O \subseteq Y$, $f\left(f^{-1}(O)\right)=O$. The straightforward proof is omitted.

Theorem 2.2. There exists a space $(X, T)$ with property $Q(1, n)$.

Proof. Let $(Y, S)$ be a space with property Qo. Let $a_{1} \in Y$, let $a_{2}, \cdots, a_{n}$ be distinct elements not in $Y$, let $X=Y \cup\left\{a_{2}, \cdots, a_{n}\right\}$, let $f: X \rightarrow Y$ such that $f$ restricted to $Y$ is the identity function and $f\left(a_{i}\right)=a_{1} ; i=2, \cdots, n$, and let $T=\left\{f^{-1}(O) \mid O \in S\right\}$. Then $T$ is a topology on $X$. Let $f^{-1}(V) \in T$ containing one of $a_{1}, \cdots, a_{n}$. Then $a_{1} \in V$ and all of $a_{1}, \cdots, a_{n}$ are in $f^{-1}(V)$. Thus every open set in $X$ containing one of $a_{1}, \cdots, a_{n}$ contains all of them and $C l\left(\left\{a_{1}\right\}\right)=\cdots=C l\left(\left\{a_{n}\right\}\right)$ and $(X, T)$ is "not- $T_{0}$ ".

Let $x \in X$ different from each of $a_{1}, \cdots, a_{n}$. Suppose there exists a $y \in X$ different from $x$ such that $C l(\{x\})=C l(\{y\})$. Then $f(x)$ and $f(y)$ are distinct elements in $Y$ and since $(Y, S)$ is $T_{0}$, there exists an open set $U$ in $Y$ containing only one of $f(x)$ and $f(y)$. Then $f^{-1}(U)$ is open in $X$ containing only one of $x$ and $y$ and $C l(\{x\}) \neq C l(\{y\})$, which is a contradiction. Thus in $X, C l(\{x\})=C l(\{y\})$ iff $x=y$. 
By definition of $T, f:(X, T) \rightarrow(Y, S)$ is continuous. Let $O \in T$. Let $U \in S$ such that $O=f^{-1}(U)$. Then $f(O)=f\left(f^{-1}(U)\right)$, which, since $f$ is onto, equals $U$. Hence $f$ is open.

For each $x \in X$, let $C_{x}$ be the element of $X_{0}$ containing $x$. Then $C_{a_{i}}=\left\{a_{1}, \cdots, a_{n}\right\} ; i=1, \cdots, n$, and for all other $x \in X, C_{x}=\{x\}$. Let $C_{x} \in X_{0}$. If $x=a_{i}$ for some $i \in\{1, \cdots, n\}$, then $C_{x} \cap Y=a_{1}$. If $x \neq a_{i}$; $i=1, \cdots, n$, then $C_{x} \cap Y=\{x\}$. Thus for each $C_{x} \in X_{0}, C_{x} \cap Y$ is a singleton set. Let $g$ be the relation in $X_{0} \times Y$ defined by $g=\left\{\left(C_{x}, y\right)\right.$ | $\left.C_{x} \cap Y=\{y\}\right\}$. If $C_{u}=C_{v}$, then $C_{u} \cap Y=C_{v} \cap Y$. Hence $g$ is a function. Since for each $x \in Y, x \in C_{x}$, then $g\left(C_{x}\right)=x$ and $g$ is onto. Let $C_{u}, C_{v} \in X_{0}$ such that $g\left(C_{u}\right)=g\left(C_{v}\right)$. If $g\left(C_{u}\right)=a_{1}$, then $C_{u}=\left\{a_{1}, \cdots, a_{n}\right\}=C_{v}$. If $g\left(C_{u}\right)=x \neq a_{1}$, then $C_{u}=C_{x}=C_{v}$. Hence $g$ is one-to-one.

Let $C_{x} \in X_{0}$. If $C_{x}=C_{a_{1}}$, then $f\left(N^{-1}\left(C_{x}\right)\right)=f\left(\left\{a_{1}, \cdots, a_{n}\right\}\right)=a_{1}=$ $g\left(C_{x}\right)$. If $C_{x} \neq C_{a_{1}}$, then $f\left(N^{-1}\left(C_{x}\right)\right)=f(\{x\})=x=g\left(C_{x}\right)$. Hence, for each $C_{x} \in X_{0}, f\left(N^{-1}\left(C_{x}\right)\right)=g\left(C_{x}\right)$. Let $O \in Q(X, T)$. Then $N^{-1}(O) \in T$ and $f\left(N^{-1}(O)\right)=g(O) \in S$. Thus $g$ is open. Let $V \in S$. Then $f^{-1}(V) \in T$ and, since $N$ is open [10], then $N\left(f^{-1}(V)\right)=g^{-1}(V) \in$ $Q(X, T)$. Hence $g$ is continuous.

Therefore $g:\left(X_{0}, Q(X, T)\right) \rightarrow(Y, S)$ is a homeomorphism. Since $(Y, S)$ is $Q o$ and $Q o$ is a topological property, then $\left(X_{0}, Q(X, T)\right)$ is $Q o$. Hence $(X, T)$ has property $Q(1, n)$ and $Q(1, n)$ exists.

Theorem 2.3. Let $(X, T)$ and $(Y, S)$ be topological spaces and let $f:(X, T) \rightarrow(Y, S)$ be a homeomorphism. Then the relation $f^{*}=\left\{\left(C_{x}\right.\right.$, $\left.\left.C_{f(x)}\right) \mid C_{x} \in X_{0}\right\}$ in $X_{0} \times Y_{0}$ is a homeomorphism. 
Proof. Since $f$ is continuous and onto, then $f^{*}$ is a continuous function from $\left(X_{0}, Q(X, T)\right)$ onto $\left(Y_{0}, Q(Y, S)\right)$ [10]. Since $f:(X, T) \rightarrow$ $(Y, S)$ is open, then $f^{*}:\left(X_{0}, Q(X, T)\right) \rightarrow\left(Y_{0}, Q(Y, S)\right)$ is open [10].

Let $C_{u}, C_{v} \in X_{0}$ such that $f^{*}\left(C_{u}\right)=f^{*}\left(C_{v}\right)$. Then $C_{f(u)}=C_{f(v)}$. Let $O \in T$ such that $u \in O$. Then $f(O)$ is open in $(Y, S), C_{u} \subseteq O$, and $C_{f(u)} \subseteq f(O)$. Since $C_{f(u)}=C_{f(v)}$, then $f(v) \in f(O)$ and $v \in O$. Thus every open set in $X$ containing $u$ contains $v$. Similarly, every open set in $X$ containing $v$ contains $u$. Thus $C l(\{u\})=C l(\{v\})$ and $C_{u}=C_{v}$. Hence $f^{*}$ is one-to-one and $f^{*}:\left(X_{0}, Q(X, T)\right) \rightarrow\left(Y_{0}, Q(Y, S)\right)$ is a homeomorphism.

Theorem 2.4. Let $Q$ be a topological property for which Qo exists and let $n$ be a natural number; $n \geq 2$. Then $Q(1, n)$ is a topological property.

Proof. Let $(X, T)$ have property $Q(1, n)$, let $(Y, S)$ be a space, and let $f:(X, T) \rightarrow(Y, S)$ be a homeomorphism. Let $a_{1}, \cdots, a_{n}$ be the $n$ distinct elements in $X$ such that $C l\left(\left\{a_{1}\right\}\right)=C l\left(\left\{a_{2}\right\}\right)=\cdots=C l\left(\left\{a_{n}\right\}\right)$ and for all other $x$ in $X, C l(\{x\})=C l(\{y\})$ iff $x=y$. Since $f:(X, T) \rightarrow(Y, S)$ is a homeomorphism, then $\left\{f\left(a_{i}\right) \mid i=1, \cdots, n\right\}$ is a set of $n$ distinct elements of $Y$ all of whose closures are equal. Let $y \in Y$ different from $f\left(a_{i}\right) ; i=1, \cdots, n$, and let $v \in Y$ different from $y$. Let $x, u \in X$ such that $f(x)=y$ and $f(u)=v$. Then $C l(\{x\}) \neq C l(\{u\})$ and there exists an open set $U$ in $X$ containing only one of $x$ and $u$. Thus $f(U)$ is open in $Y$ containing only one of $x$ and $v$ and $C l(\{y\}) \neq C l(\{v\})$.

Since $(X, T)$ has property $Q(1, n)$, then $\left(X_{0}, Q(X, T)\right)$ has property Qo and since $f:(X, T) \rightarrow(Y, S)$ is a homeomorphism, then, by Theorem 2.3, $\left(Y_{0}, Q(Y, S)\right)$ has property $Q$ o. Hence $(Y, S)$ has property $Q(1, n)$. Therefore $Q(1, n)$ is a topological property. 
Corollary 2.1. Let $Q$ be a topological property for which Qo exists and let $m$ and $n$ be natural numbers greater than or equal to 2 with $m<n$. Then $Q(1, m)$ and $Q(1, n)$ are topologically distinct topological properties.

Definition 2.2. Let $Q$ be a topological property such that $Q o$ exists. A space $(X, T)$ has property $Q N O$ iff $(X, T)$ is "not- $T_{0}$ " and $\left(X_{0}, Q(X, T)\right)$ has property $Q$.

Theorem 2.5. Let $Q$ be a topological property for which Qo exists. Then $Q N O$ is a topological property.

Proof. Let $(X, T)$ have property $Q N O$, let $(Y, S)$ be a homeomorphic image of $(X, T)$, and let $f:(X, T) \rightarrow(Y, S)$ be a homeomorphism. Since $(X, T)$ is "not- $T_{0}$ " and "not- $T_{0}$ " is a topological property, then $(Y, S)$ is "not- $T_{0}$ ". Since $(X, T)$ has property $Q N O$, then $\left(X_{0}, Q(X, T)\right)$ has property $Q o$. Since $Q o$ is a topological property, then, by Theorem 2.3, $\left(Y_{0}, Q(Y, S)\right)$ has property $Q o$. Thus $Q N O$ is a topological property.

Theorem 2.6. Let $Q$ be a topological property for which Qo exists. Then there are infinitely many topologically distinct topological properties that are QNO.

Proof. Let $n$ be a natural number $n \geq 2$ and let $(X, T)$ be a space with property $Q(1, n)$. Then $(X, T)$ has property $Q N O$ and $Q N O$ exists. Since, by Corollary 2.1, for distinct natural numbers $m$ and $n$ each greater than or equal to $2, Q(1, m)$ and $Q(1, n)$ are topologically distinct topological properties, there are infinitely many topologically distinct topological properties that are $Q N O$.

Theorem 2.7. Let $Q$ be a topological property for which Qo exists and let $W$ be a topological property such that for each space with property $W$ its $T_{0}$-identification space has property $Q$ o. Then $W=(Q o$ or $Q N O)$. 
Proof. Since $L$ is the least topological property, then $W=$ $(W$ and $L)=\left(W\right.$ and $\left(T_{0}\right.$ or "not- $\left.T_{0} "\right)=\left(W o\right.$ or $\left(W\right.$ and "not $\left.\left.-T_{0} "\right)\right)$. Let $(X, T)$ be a space with property $W$. If $(X, T)$ is $T_{0}$, then $W=W o$, and, since a space is $T_{0}$ iff the natural map $N:(X, T) \rightarrow\left(X_{0}, Q(X, T)\right)$ is a homeomorphism, then $\left(X_{0}, Q(X, T)\right)$ has property Wo, which implies $\left(X_{0}, Q(X, T)\right)$ is both $W o$ and $Q o$ and $W o=Q o$. Thus consider the case that $W=\left(W\right.$ and "not- $T_{0}$ " $)$. Then $(X, T)$ is "not- $T_{0}$ " and $\left(X_{0}, Q(X, T)\right)$ has property $Q o$, which implies $(X, T)$ has property $Q N O$. Thus $W=(Q o$ or $Q N O)$.

Corollary 2.2. Let $Q$ be a topological property for which Qo exists. Then (Qo or QNO) is the least of all topological properties $W$ for which the $T_{0}$-identification space of each space with property $W$ has property Qo.

Theorem 2.8. Let $Q$ be a topological property for which Qo exists and let $(X, T)$ be a space. Then $(X, T)$ has property (Qo or QNO) iff $\left(X_{0}, Q(X, T)\right)$ has property (Qo or $\left.Q N O\right)$.

Proof. Suppose $(X, T)$ has property (Qo or $Q N O)$. Then $\left(X_{0}, Q(X, T)\right)$ has property $Q o$, which implies its $T_{0}$-identification space has property $Q o$ and $\left(X_{0}, Q(X, T)\right)$ has a topological property for which its $T_{0}$-identification space is $Q o$. Thus $\left(X_{0}, Q(X, T)\right)$ has property ( $Q o$ or $Q N O)$.

Conversely, suppose $\left(X_{0}, Q(X, T)\right)$ has property (Qo or $\left.Q N O\right)$. Since $\left(X_{0}, Q(X, T)\right)$ is $T_{0}$, then $\left(X_{0}, Q(X, T)\right)$ has property $(Q o \text { or } Q N O)_{o}=Q o$ and $(X, T)$ has property (Qo or $Q N O)$.

Using the fact that the $T_{0}$-identification $P$ properties are precisely those topological properties $Q$ that are weakly $Q o$, gives the next result. 
Corollary 2.3. Let $Q$ be a topological property for which Qo exists. Then $(Q o$ or $Q N O)$ is a $T_{0}$-identification $P$ property, (Qo or $\left.Q N O\right)=$ weakly $(\mathrm{Qo} \text { or } \mathrm{QNO})_{\mathrm{O}}=$ weakly $\mathrm{Qo}$ and $(\mathrm{Qo}$ or $\mathrm{QNO})$ is a weakly Po property.

Corollary 2.4. Let $Q$ be a topological property for which Qo exists. Then (Qo or QNO) is a topological property for which both $T_{0}$ and "not- $T_{0}$ " exist and $\left(Q o\right.$ or "not- $T_{0}$ ”) $=$ Qo.

In the 2016 paper [6], it was proven that for a topological property $Q$ for which weakly $Q o$ exists, weakly $Q o=\left(Q o\right.$ or (weakly $Q o$ and“not- $T_{0}$ ”)), which is used with the results above to get the next result.

Theorem 2.9. Let $Q$ be a topological property for which both Qo and ( $Q$ and "not- $T_{0}$ ") exist. Then $Q$ is a $T_{0}$-identification $P$ property, $Q N O=\left(Q\right.$ and "not $\left.-T_{0} "\right)$, and $Q=$ weakly $Q o=\left(Q o\right.$ or $\left(Q\right.$ and "not $\left.\left.-T_{0} "\right)\right)$.

Proof. Let $(X, T)$ be a space. Since (Qo or $Q N O)$ is a $T_{0}$-identification $P$ property and weakly $P_{0}$, then $(X, T)$ has property $(Q o$ or $Q N O)$ iff $\left(X_{0}, Q(X, T)\right)$ has property (Qo or $\left.Q N O\right)$ iff $\left(X_{0}, Q(X, T)\right)$ has property $(Q o$ or $Q N O) o=Q_{0}$ iff $\left(X_{0}, Q(X, T)\right)$ has property $Q$ iff $(Q o$ or $Q N O ")=Q$ iff $(X, T)$ has property $Q$. Thus $Q$ is a $T_{0}$-identification $P$ property and $Q=$ weakly $Q o$. Hence $Q=(Q o$ or $(Q$ and "not- $T_{0}$ ") $)=$ weaklyQo $=(Q o$ or $Q N O)$ and $Q N O=((Q o$ or $Q N O)$ $\backslash Q o)=\left(\left(Q o\right.\right.$ or $\left(Q\right.$ and "not- $\left.\left.\left.T_{0} "\right)\right) \backslash Q o\right)=\left(Q\right.$ and "not- $\left.T_{0} "\right)$.

Theorem 2.10. $\left\{P \mid P\right.$ is a $T_{0}$-identification $Q$ property $\}=\{P \mid P$ is a topological property and weakly Po exists $\}=\{P \mid P$ is a topological property and both Qo and ( $Q$ and "not- $T_{0}$ ") exist\}.

Proof. By the results above, $\left\{P \mid P\right.$ is a $T_{0}$-identification $Q$ property $\}=\{P \mid P$ is a topological property and weakly $P o$ exists $\}$. 
Let $Q \in\{P \mid P$ is a topological property and both $Q o$ and $(Q$ and "not- $T_{0}$ ") exist $\}$. Then $Q o$ exists and, by Theorem $2.9, Q \in\{P \mid P$ is a topological property and weakly Qo exists\}.

Let $Q \in\{P \mid P$ is a topological property and weakly $P o$ exists $\}$. Since weakly $Q o$ exists, then $Q_{o}$ is a weakly $P o$ property and $Q o$ exists. Then, by Theorem 2.9, $Q \in\{P \mid P$ is a topological property and both $Q o$ and $\left(Q\right.$ and "not- $T_{0}$ ") exist $\}$.

Hence, $\left\{P \mid P\right.$ is a $T_{0}$-identification $Q$ property $\}=\{P \mid P$ is a topological property and weakly $Q o$ exists $\}=\{P \mid P$ is a topological property and both $Q o$ and ( $Q$ and "not- $T_{0}$ ”) exist\}.

Corollary 2.5. $\left\{Q \mid Q\right.$ is a $T_{0}$-identification P property $\}=\{Q o \mid Q$ is a topological property and Qo is a weakly Po property $\}=\{Q o \mid Q$ is a topological property and Qo exists\}.

\section{The Complete Characterization of $T_{0}$-identification Space Properties}

Theorem 3.1. $\left\{P \mid P\right.$ is a $T_{0}$-identification space property $\}=\{P o \mid P$ is a topological property and Po exists $\}=\left\{P \mid P\right.$ is a $T_{0}$-identification $Q$ property\}.

Proof. Let $Q$ be a $T_{0}$-identification space property. Let $(X, T)$ be a space for which $\left(X_{0}, Q(X, T)\right)$ has property $Q$. Then $\left(X_{0}, Q(X, T)\right)$ has property $Q o$. Thus $Q o$ exists and $\left\{P \mid P\right.$ is a $T_{0}$-identification space property $\} \subseteq\{P o \mid P$ is a topological property and $P o$ exists $\}$.

Suppose $Q$ is a topological property for which $Q o$ exists. Then, by the results above, $Q o$ is a weakly $P o$ property, which is, by definition, a $T_{0}$-identification space property. Thus $\{P o \mid P$ is a topological property and $P o$ exists $\} \subseteq\left\{P \mid P\right.$ is a $T_{0}$-identification space property $\}$. 
Thus, by the results above, $\left\{P \mid P\right.$ is a $T_{0}$-identification space property $\}=\{P o \mid P$ is a topological property and $P o$ exists $\}=\{P \mid P$ is a $T_{0}$-identification $Q$ property\}.

As established above, the introduction and investigation of $T_{0}$-identification spaces in 1936 [12] added an important, strong tool for use in the continued investigation and expansion of topology. The 1936 characterization of metrizable [12] and the 1975 characterization of $T_{2}$ [11] were important papers that led to the introduction and investigation of weakly Po spaces and properties leading to the discovery and use of never before imagined properties, which now includes the never before imagined reality that the characterizations of metrizable and $T_{2}$ are special cases of all topological properties $P$ for which $P o$ exists.

\section{References}

[1] A. Davis, Indexed systems of neighborhoods for general topological spaces, Amer. Math. Monthly 68 (1961), 886-893.

[2] C. Dorsett, New characterizations of separation axioms, Bulletin of the Calcutta Mathematical Society 99(1) (2007), 37-44.

[3] C. Dorsett, Characterizations of spaces using $T_{0}$-identification spaces, Kyungpook Mathematical Journal 17(2) (1977), 175-179.

[4] C. Dorsett, Weakly $P$ properties, Fundamental J. Math. Math. Sci. 3(1) (2015), 83-90.

[5] C. Dorsett, $T_{0}$-identification $P$ and weakly $P$ properties, Pioneer Journal of Mathematics and Mathematical Sciences 15(1) (2015), 1-8.

[6] C. Dorsett, Weakly $P$ corrections and new fundamental topological properties and facts, Fundamental Journal of Mathematics and Mathematical Sciences 5(1) (2016), 11-20.

[7] C. Dorsett, Another important use of "not- $P$ ", where $P$ is a topological property, accepted by Pioneer Journal of Mathematics and Mathematical Sciences.

[8] C. Dorsett, Pluses and needed changes in topology resulting from additional properties, Far East Journal of Mathematical Sciences 101(4) (2017), 803-811. 
[9] C. Dorsett, New properties tools and changes for subspace properties and singleton set spaces, Pioneer Journal of Mathematics and Mathematical Sciences 17(2) (2016), 79-85.

[10] C. Dorsett, $T_{0}$-identification spaces and $R_{1}$ spaces, Kyungpook Mathematical Journal 18(2) (1978), 167-174.

[11] W. Dunham, Weakly Hausdorff spaces, Kyungpook Math. J. 15(1) (1975), 41-50.

[12] M. Stone, Application of Boolean algebras to topology, Mat. Sb. 1 (1936), 765-771. 